\title{
Perfusion-CT: A Non-Invasive Tool to Monitor Regional Brain Perfusion in Traumatic Brain Injury Patients and Therapies Impacting it
}

\author{
Max Wintermark*,1 and Jean-Francois Soustiel ${ }^{2}$ \\ ${ }^{I}$ Department of Radiology, Neuroradiology Division, University of Virginia, Charlottesville, Virginia, USA \\ ${ }^{2}$ Acute Brain Injury Research Laboratory, Department of Neurosurgery, Rambam Medical Center, Faculty of Medicine, \\ Technion - Israel Institute of Technology, Haifa, Israel
}

\begin{abstract}
There is a growing body of evidence suggesting imaging biomarkers to select patients with traumatic brain injury (TBI) for therapies, and to monitor the effects of these treatments. In this setting, advanced neuroimaging techniques such as perfusion-CT (PCT) can offer more clinically relevant pathophysiologic information than purely structural imaging such as conventional noncontrast CT. Patients with altered brain PCT results might be considered for more aggressive and early treatment to prevent intracranial hypertension, whereas patients with preserved brain perfusion might benefit from less invasive treatment. From a clinical trial perspective, better stratification of the patient population, and the use of advanced neuroimaging as a biomarker for response to intervention, could be expected to significantly reduce the number of patients required to assess a treatment effect and, therefore, expedite the clinical trial process. This article reviews the fundamental of perfusion-CT technique and its application to TBI.
\end{abstract}

Keywords: Traumatic brain injury, prognosis, imaging, perfusion-CT.

\section{INTRODUCTION}

Brain perfusion in head trauma patients has been imaged using different techniques, including stable Xenon-CT [1,2] and single photon emission computed tomography (SPECT) $[3,4]$. These brain perfusion imaging techniques suffer different drawbacks. SPECT does not afford quantitative results, but only a qualitative comparison between the right and left hemispheres [5]. On the other hand, stable Xenon$\mathrm{CT}$ is quantitatively accurate, but requires specialized and expensive equipment. A typical study is relatively long, approximately 10 minutes. Side effects, such as respiratory rate decrease, headaches, nausea and vomiting, as well as convulsions, are observed in $4.4 \%$ of patients [6]. Consequently, stable Xenon-CT is difficult to perform in severe trauma patients in the emergency settings.

Recently, perfusion-CT (PCT) has been introduced as a simple imaging technique to be used in routine clinical practice [7, 8]. PCT involves dynamic acquisition of sequential $\mathrm{CT}$ slices on a cine mode during intravenous administration of non-ionic iodinated contrast material. We believe that PCT technique can be implemented in all hospital institutions equipped with CT units, which are usually available around the clock and 7 days a week. It necessitates neither specialized technologists nor extra material, but only requires dedicated post-processing software. It affords realtime post-processing, with a complete set of parametric maps typically generated within 5 minutes of completing data acquisition. PCT provides quantitatively accurate assessment

*Address correspondence to this author at the University of Virginia, Department of Radiology, Neuroradiology Division, 1215 Lee Street, Box 800170, Charlottesville, VA 22908, USA; Tel: 434-243-9312;

Fax: 434-982-5753; E-mail: Max.Wintermark@ virginia.edu of brain perfusion: its results have been validated by comparison with stable xenon CT [9] and PET [10].

PCT can easily be performed as a complement to conventional noncontrast and contrast-enhanced cerebral CT, and does not interfere with the contrast-enhanced thoracoabdominal CT survey performed in severe trauma patients [11]. In our institution, as in most trauma centers, we obtain contrast-enhanced chest, abdomen and pelvis CT routinely in severe trauma patients in order to rule out aortic injuries. The contrast material administration is performed even in obtunded patients unable to report about possible previous contrast reactions or without knowing about the renal function, because the risk associated with these conditions is outweighed significantly by the risk of a missed traumatic aortic injury. The dose of contrast material $(40-50 \mathrm{ml})$ added for the PCT is minor compared to the dose used for the chest, abdomen and pelvis (100-120 ml).

\section{BRAIN PERFUSION-CT TECHNIQUE: DATA AC- QUISITION}

PT scans are obtained at our institution by using a multidetector row scanner. Each PCT study involves successive gantry rotations performed in cine mode during intravenous administration of iodinated contrast material. Images are acquired and reconstructed at a temporal sampling rate of 1 image per second for the first 37 seconds and 1 image every 2 seconds for the next 33 seconds. Additional gantry rotations are obtained at 90, 120, 150, 180, 210 and 240 seconds. Acquisition parameters are $80 \mathrm{kVp}$ and $100 \mathrm{mAs}$. One or two PCT series are typically obtained following the noncontrast $\mathrm{CT}$ of the brain and prior to the $\mathrm{CT}$ of the cervical spine and/or CT-angiogram of the aorta (PCT can be used as a test bolus to determine the optimal timing for the CTangiogram of the aorta). At each PCT level, eight 5-mm- 
thick slices are assessed. CT gantry tilt for all perfusion CT (and noncontrast CT) studies is selected parallel to the hard palate. The PCT series is obtained above the orbits, at the level of the third ventricle and the basal ganglia, and sometimes a second PCT series is obtained immediately superior to the first PCT slab, above the lateral ventricles. For each PCT series, a $40 \mathrm{ml}$ bolus of iohexol (Omnipaque, Amersham Health, Princeton, NJ; $300 \mathrm{mg} / \mathrm{ml}$ of iodine) are administered into an antecubital vein using a power injector at an injection rate of $5 \mathrm{ml}$ per second for all patients. CT scanning is initiated 7 seconds after start of the injection of the contrast bolus.

\section{BRAIN PERFUSION-CT TECHNIQUE: DATA PROC- ESSING}

PCT data is analyzed utilizing commercially available PCT software (Brain Perfusion, Philips Healthcare, Cleveland, $\mathrm{OH}, \mathrm{USA})$. This software relies on the central volume principle, which is the most accurate for low injection rates of iodinated contrast material. The software obtains mathematical descriptions of the time-density curves for each pixel, by applying least mean squares curve fitting, after correcting for motion and noise reduction through an anisotropic, edge-preserving spatial filter. A closed-form deconvolution is then applied to calculate the mean transit time (MTT) map. The deconvolution operation requires a reference arterial input function (most often within the anterior cerebral artery), automatically selected by the PCT software within a region of interest (ROI) drawn by the user. The cerebral blood volume (CBV) map is calculated from the area under the time-density curves compared to a similarly obtained venous reference curve. The reference "vein" actually needs to be the pixel with the largest area under its contrast-enhancement curve. As such, it must be selected at the center of the largest vascular structure perpendicular to the PCT slices. These requirements are usually met by pixels at the center of the superior sagittal sinus. However, in some instances, other venous structures, or even the supraclinoid internal carotid arteries, can be appropriate "veins" for PCT processing purposes. CBF is calculated as the CBV/MTT ratio.

\section{BRAIN PERFUSION-CT IN TBI PATIENTS}

\section{Assessment of Intracranial Traumatic Lesions}

PCT affords insight into regional brain perfusion alterations due to head trauma, with the major advantage of being able to detect regional heterogeneity [11]. For instance, PCT can quantify the effect of an extra-axial blood collection (epidural or subdural hematoma) on the perfusion of the adjacent brain parenchyma (Fig. 1). PCT has a higher sensitivity for the diagnosis of cerebral contusions (Fig. 2) when compared to admission non-contrast cerebral CT, with a sensitivity reaching $87.5 \%$ versus $39.6 \%$ for conventional CT

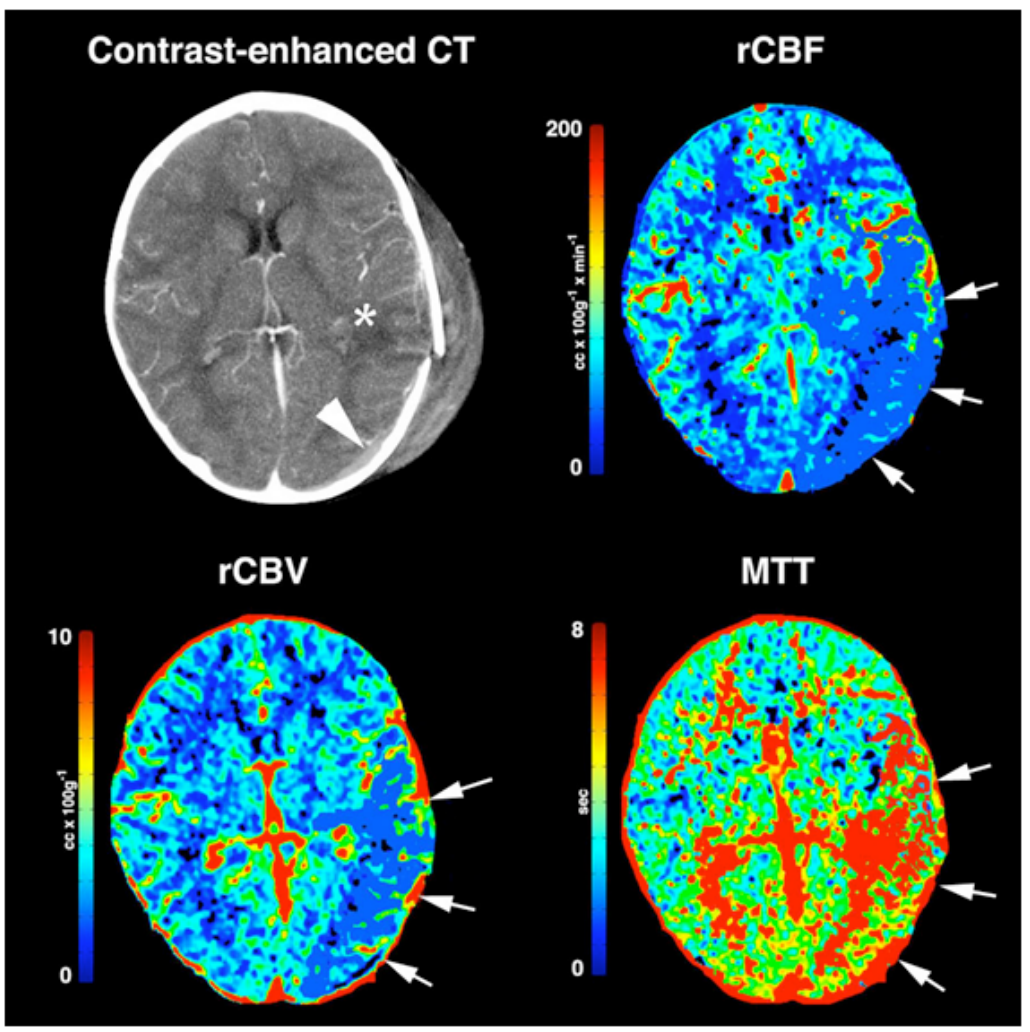

Fig. (1). Patient fallen from a 6-m height, admitted with a Glasgow Coma Scale score of 9. Neurological examination in the emergency room reveals an asymmetry of tone and deep tendon reflex involving both right upper and lower limbs. Admission contrast-enhanced cerebral CT demonstrates a displaced left parietal skull fracture, associated with a large cephalhematoma. A small left parieto-occipital epidural hematoma (white arrowhead) and a small contusion area (white star) could also be identified on the conventional CT images. PCT demonstrates a much wider area of brain perfusion compromise (white arrows) and an involvement of the whole left temporal and parietal lobes, the latter showing increased mean transit time (MTT) and decreased regional cerebral blood flow (rCBF) and volume (rCBV). Thus, PCT affords a better understanding of the neurological examination findings on admission than conventional CT. 


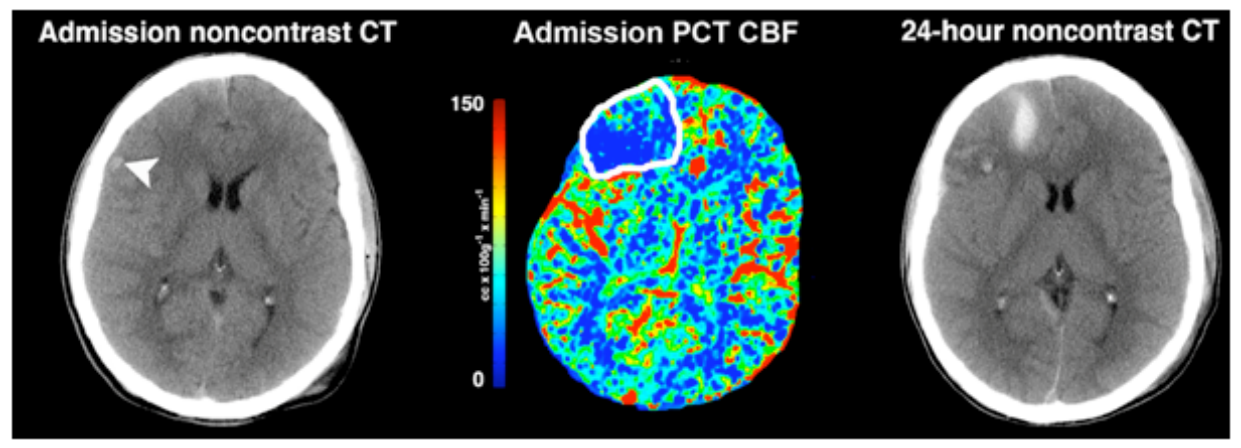

Fig. (2). 20-year-old male patient involved in a high-speed traffic accident. On admission, noncontrast cerebral CT features only a small hemorrhagic focus in the right frontal operculum (arrowhead). On the other hand, admission perfusion-CT shows a more extensive area of brain perfusion abnormality, involving most of the right frontal lobe (arrows). This area is characterized by low low rCBF values. Noncontrast cerebral CT obtained 24 hours after admission has become positive for hemorrhagic contusion in the exact zone demonstrated as abnormal by admission perfusion-CT.

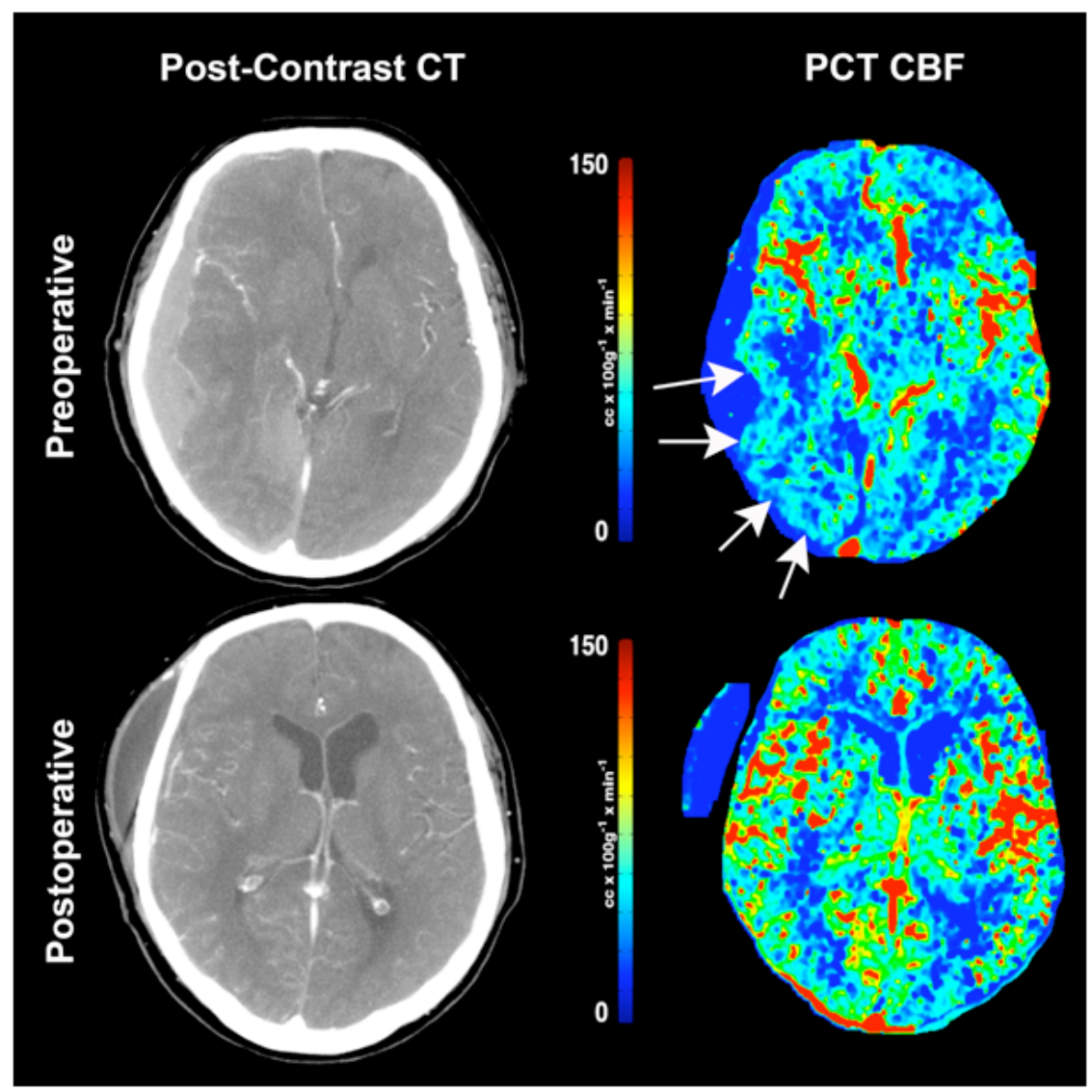

Fig. (3). Details of perfusion CT in the right frontal region in a TBI patient with a cerebral contusion. In this case as in most patients, the traumatic lesion is characterized by a mixed "salt and pepper" appearance. Analysis of several similarly hypodense regions of interest (ROIs 4, 5, 6 and 7) by PCT revealed an impressive disparity in terms of cerebral blood volume and cerebral blood flow, indicative of a similar variability in terms of tissue viability that could not be possibly predicted by plain CT alone. On the contrary, hyperdense regions are more homogenously correlating with hemorrhagic necrosis.

Upper: Superimposition of non-contrast CT image with the various ROIs selected for analysis. Lower: PCT parameters (cerebral blood volume-CBV; cerebral blood flow - CBF; mean transit time - MTT; time to peak - TTP) are indicated for each ROI with its reference number.

[11-13]. This increased accuracy of PCT to define cerebral contusion may be of critical importance for in identification of non-hemorrhagic contusions on initial non-contrast CT. In mixed lesions often characterized by a "salt-and-pepper" appearance, the non hemorrhagic hypodense component of the cerebral contusion contusions cannot be easily differentiated from perilesional edema, making the delineation of the true extent of the lesion challenging and often inaccurate. In these situations, analysis of PCT parameters within and around the lesion may be particularly useful (Fig. 3). Furthermore, cerebral contusions are characterized by their specific capacity to enlarge and turn into large hemorrhagic lesions and have been shown to represent the most frequent indication for delayed surgical intervention [14]. Accord- 
ingly, cerebral contusions represent a common surgical dilemma by associating a potentially growing mass mixed with presumably viable tissue, a distinction of critical importance whenever surgery is considered, especially in neurologically eloquent areas. In a recent study, PCT proved to be a useful tool for differentiating necrotic from viable tissue suggesting therefore its implementation for surgical planning in neurologically eloquent areas [13]. Finally, early PCT proved to better correlate with final tissue outcome than non-contrast CT, a fact of importance for precocious identification of patients at high risk of lesion growth [13].

\section{Characterization of Post Traumatic Cerebral Hemody- namics}

PCT results show specific patterns, linked to cerebral edema and intracranial hypertension $[14,15]$. Finally, the number of arterial territories with "low" rCBV values on PCT is an independent of the functional outcome, and this as early as on admission [11]. The potential repercussions of PCT on the clinical management of severe head trauma patients remain to be evaluated.

\section{FUTURE PERSPECTIVES}

\section{PCT as a Classification Tool for Categorization of TBI Patients}

Several imaging classification and prognostication schemes have been utilized in TBI research, including tools such as the Marshall and Rotterdam scores, which are based on acute CT scan findings [16, 17]. These tools characterize injuries in relatively broad categories and have been shown to have significant prognostic value with respect to outcome $[16,17]$. However, these prognostic CT models typically focus on severely injured patients; they do not explain how specific deficits related to regional damage may affect outcome, and they do not include PCT, which may be more sensitive to physiologic changes as part of their predictive data input. The use of modern imaging technology, which can offer more detailed pathophysiologic information, could have a major impact on the choice of acute treatments for patients with TBI, and on the monitoring of their effects (Fig. 4). Patients with altered brain PCT results might be considered for more aggressive and early treatment to pre-

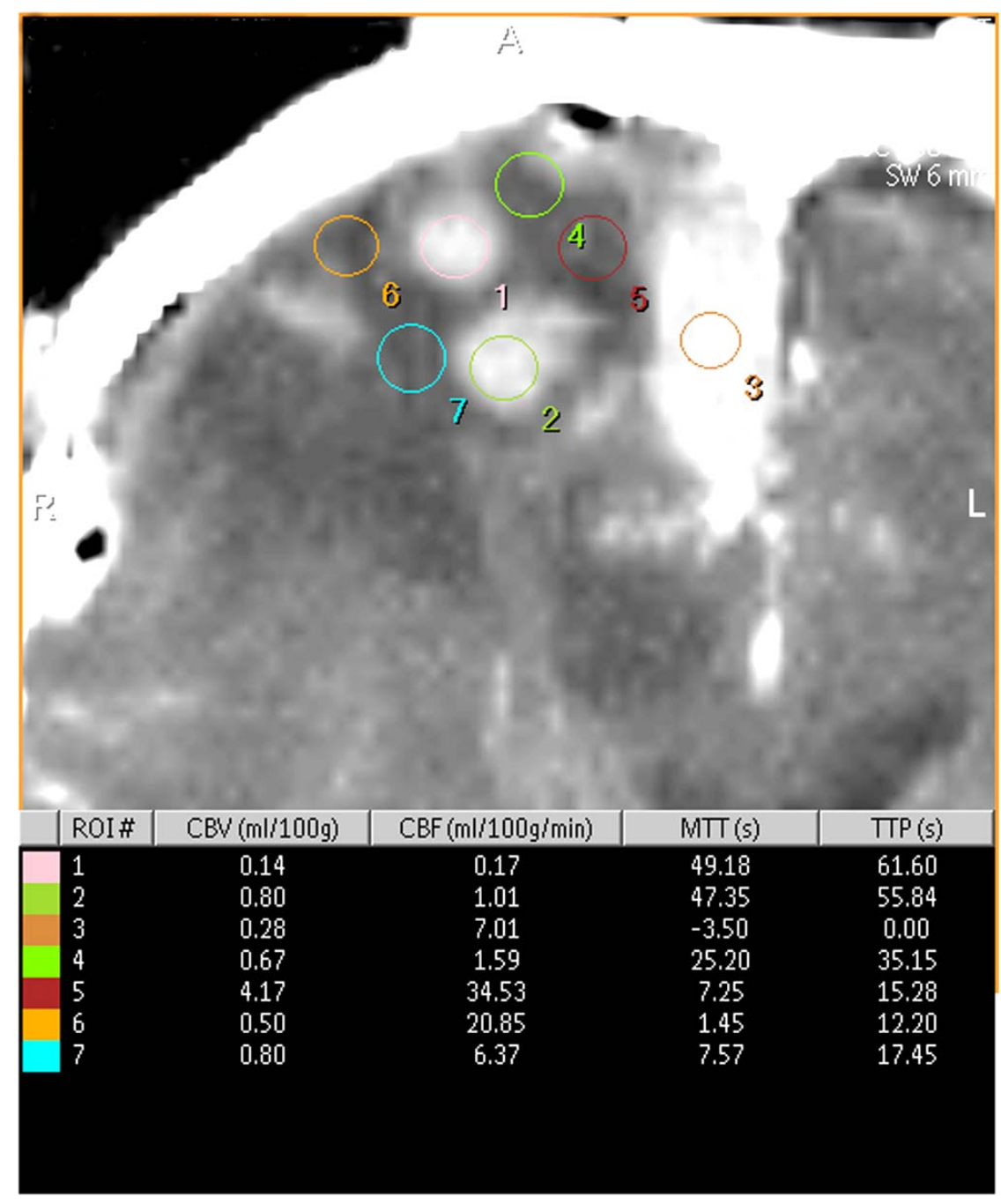

Fig. (4). Perfusion-CT studies obtained in the same patient before and after surgical evacuation of a right subdural hematoma. The initial perfusion-CT study shows the hemodynamical significance of the subdural hematoma, which compromises the perfusion of the adjacent cortical gray matter. After evacuation of the right subdural hematoma, normal brain perfusion was restored. This example illustrates the ability of perfusion-CT to monitor the effect of treatment in TBI patients. 
vent intracranial hypertension, whereas patients with preserved brain perfusion might benefit from less invasive treatment. From a clinical trial perspective, better stratification of the patient population, and the use of PCT as a biomarker or surrogate endpoint for response to intervention, could be expected to significantly reduce the number of patients required to assess a treatment effect and, therefore, expedite the clinical trial process.

\section{Assessment of Cerebral Circulatory Arrest}

Severe TBI account for approximately $40 \%$ of all trauma deaths and as such patients with severe irreversible brain damage may eventually appear as potential donors for organ transplantation. For obvious medical, ethical and medicolegal reasons, the diagnosis of brain death is a matter of constant debate, trying to integrate the need for an early decision before multi-organ failure develops and precludes the use of already damaged organs on one hand and, on the other hand, the critical though often lengthy though process of diagnosis. Among various criteria used for the diagnosis of brain death, cerebral circulatory arrest evidenced by cerebral angiography has been recognized as a reliable method though its application is limited by obvious logistic considerations. CTangiography has been advocated as an alternative diagnostic route although both false positive [18] and false negative studies have been reported [19], precluding the use of CT angiography alone for diagnosis of brain death. In these situations, PCT may be appear as a valuable adjunct tool by showing absence of cerebral blood flow in the entire brain parenchyma [19].

\section{REFERENCES}

[1] Adelson PD, Clyde B, Kochanek PM, Wisniewski SR, Marion DW, Yonas H. Cerebrovascular response in infants and young children following severe traumatic brain injury: a preliminary report. Pediatr Neurosurg 1997; 26: 200-7.

[2] Verweij BH, Muizelaar JP, Vinas FC. Hyperacute measurement of intracranial pressure, cerebral perfusion pressure, jugular venous oxygen saturation, and laser Doppler flowmetry, before and during removal of traumatic acute subdural hematoma. J Neurosurg 2001; 95: 569-72.

[3] Bavetta S, Nimmon CC, White J, et al. A prospective study comparing SPET with MRI and CT as prognostic indicators following severe closed head injury. Nucl Med Commun 1994; 15: 961-8.

[4] Nedd K, Sfakianakis G, Ganz W, et al. 99mTc-HMPAO SPECT of the brain in mild to moderate traumatic brain injury patients: compared with CT--a prospective study. Brain Inj 1993; 7: 469-79.
[5] Peters AM, Gunasekera RD, Henderson BL, et al. Noninvasive measurement of blood flow and extraction fraction. Nucl Med Commun 1987; 8: 823-37.

[6] Latchaw RE, Yonas H, Pentheny SL, Gur D. Adverse reactions to xenon-enhanced CT cerebral blood flow determination. Radiology 1987; 163: 251-54.

[7] Wintermark M, Reichhart M, Cuisenaire O, et al. Comparison of admission perfusion computed tomography and qualitative diffusion- and perfusion-weighted magnetic resonance imaging in acute stroke patients. Stroke 2002; 33: 2025-31.

[8] Wintermark M, Reichhart M, Thiran JP, et al. Prognostic accuracy of cerebral blood flow measurement by perfusion computed tomography, at the time of emergency room admission, in acute stroke patients. Ann Neurol 2002; 51: 417-32.

[9] Wintermark M, Thiran JP, Maeder P, Schnyder P, Meuli R. Simultaneous measurement of regional cerebral blood flow by perfusion $\mathrm{CT}$ and stable xenon CT: a validation study. Am J Neuroradiol 2001; 22: 905-14.

[10] Kudo K, Terae S, Katoh C, et al. Quantitative cerebral blood flow measurement with dynamic perfusion CT using the vascular-pixel elimination method: comparison with $\mathrm{H} 2(15) \mathrm{O}$ positron emission tomography. Am J Neuroradiol 2003; 24: 419-26.

[11] Wintermark M, Van Melle G, Schnyder P, et al. Admission perfusion CT: prognostic value in patients with severe head trauma. Radiology 2004; 232: 211-20.

[12] Depreitere B, Aviv R, Symons S, et al. Study of perfusion in and around cerebral contusions by means of computed tomography. Acta Neurochir Suppl 2008; 102: 259-62.

[13] Soustiel JF, Mahamid E, Goldsher D, Zaaroor M. Perfusion-CT for early assessment of traumatic cerebral contusions. Neuroradiology 2008; 50: 189-96.

[14] Wintermark M, Chiolero R, Van Melle G, et al. Relationship between brain perfusion computed tomography variables and cerebral perfusion pressure in severe head trauma patients. Crit Care Med 2004; 32: 1579-87.

[15] Wintermark M, Chiolero R, Van Melle G, et al. Cerebral vascular autoregulation assessed by perfusion-CT in severe head trauma patients. J Neuroradiol 2006; 33: 27-37.

[16] Maas AI, Hukkelhoven CW, Marshall LF, Steyerberg EW. Prediction of outcome in traumatic brain injury with computed tomographic characteristics: a comparison between the computed tomographic classification and combinations of computed tomographic predictors. Neurosurgery 2005; 57: 1173-82.

[17] Marshall LF, Marshall SB, Klauber MR, et al. The diagnosis of head injury requires a classification based on computed axial tomography. J Neurotrauma 1992; 9(Suppl 1): S287-S292.

[18] Greer DM, Strozyk D, Schwamm LH. False positive CT angiography in brain death. Neurocrit Care 2009; 11: 272-75.

[19] Escudero D, Otero J, Marques L, et al. Diagnosing brain death by CT perfusion and multislice CT angiography. Neurocrit Care 2009; 11: 261-71.

(C) Wintermark and Soustiel; Licensee Bentham Open.

This is an open access article licensed under the terms of the Creative Commons Attribution Non-Commercial License (http://creativecommons.org/licenses/by-nc/3.0/) which permits unrestricted, non-commercial use, distribution and reproduction in any medium, provided the work is properly cited. 